DOE/ER/ 14223--TI

DE92 040558

\title{
PARTICLE PRESSURES IN FLUIDIZED BEDS
}

\author{
Grant \# DE-FG03-91ER14223
}

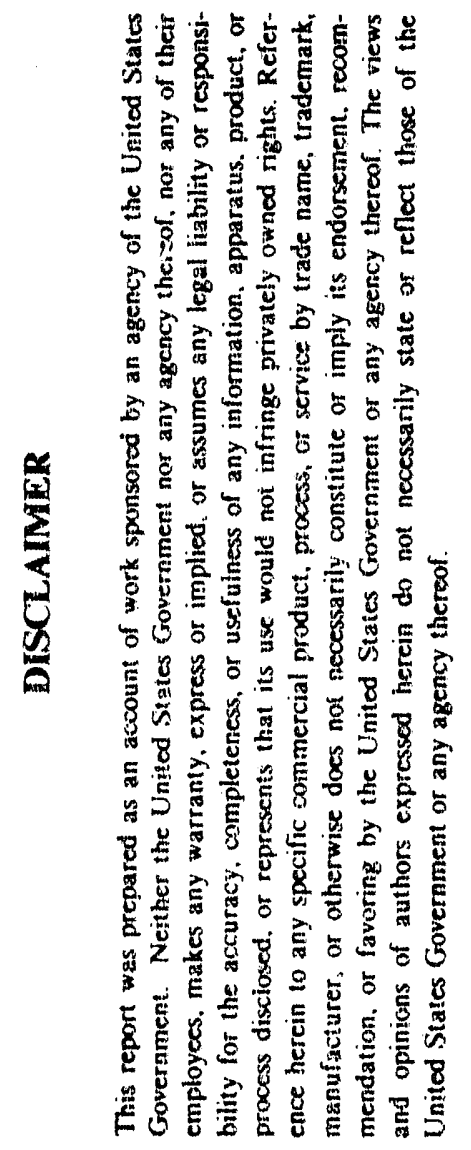

\author{
FIRST YEAR ANNUAL REPORT
}

1992

Charles S. Campbell

Khurram Rabrnan

Xiao $\mathrm{Hu}$

Cheragahen Jin

and

Alexander V. Fotapov

Department of Mechanical Engineering

University of Southern California

Los Angeles, Ca. 90089-1453 


\section{SUMMARY}

This is an experimental project to make detailed measurements of the particle pressures generated in fluidized beds. The focus lies in two principle areas: (1) the particle pressure distribution around single bubbles rising in a two-dimensional gas-fluidized bed and (2) the particle pressures measured in liquid-fluidized beds.

This first year has largely been to constructing the experiments. The design of the particle pressure probe has been improved and tested. A two-dimensional gas-fluidized bed has been constructed in order to measure the particle pressure generated arcund injected bubbles. The probe is also being adapted to work in a liquid fluidized bed. Finally, a two-dimensional liquid fluidized bed is also under construction.

Preliminary measurements show that the majority of the particle pressures are generated in the wake of a bubble. However, the particle pressures generated in the liquid bed appear to be extremely small.

Finally, while not directly associated with the particle pressure studies, some NERSC supercomputer time was granted alongside this project. This is being used to make large scale computer simulation of the flow of granular materials in hoppers. 


\section{TABLE OF CONTEN'TS}

$\begin{array}{lll}1.0 & \text { Introduction } & 4\end{array}$

2.0 Improved Particle Pressure Transducer 6

2.1 New Transducer Design 6

2.2 Archetypical Particle Pressure Measurernents 10

3.0 Particle Pressure Measurements Around Single Bubbles in a Two-Dimensional Gas-Fluidized Bed 14

4.0 Particle Pressure Measurements in Water Fluidized Beds 19

5.0 Computer Sirnulation of Hopper Flows 24

$\begin{array}{lll}6.0 & \text { References } & 28\end{array}$ 


\subsection{INTRODUCTION}

The particle pressure may be thought of as the force per unit area exerted by the particulate phase of a multiphase mixture and, as such, reflects the total momentum tratsport that can be attributed to the motion of particles and their interactions. It has a direct analog in the kinetic theory of gases in which the pressure acting on a surface is visualized as a result of the impacts of molecules. The same picture can be applied to particle-fluid situations with the particles taking the place of molecules. The only difference between the two cases is that solid particles may, in addition to shortduration collisional impacts, transmit a force via long duration contacts. (E.g. the weight of a particle, or an assembly of particles, resting on a surface).

The difficulty in measuring the particle pressure is that the total pressure exerted on a surface - the pressure that would be measured with a star Jard flush mounted pressure transducer - is the sum of the particle pressure and the pressure exerted by the fluid that resides in the interstices between the particles. Furthermore, in many cases for example, fluidized beds or slurry flows - in which the motion is driven by fluid pressure - the particle pressure may be a small fraction of the total. Conceptually, such a measurement is not complicated, nor is the measurement terribly difficult. Essentially all one has to do is measure the total force acting on a surface and then let that fraction due to the fluid pressure balance itself out. An erlier paper, Campbell \& Wang (1990) described a very simple transducer for this purpose. That probe consisted of a solid diaphragm flush mounted into the wall. Small holes on either side of the diaphragm admit fluid, but no particles into a chamber behind the diaphragm. The face of the diaphragm experiences the total pressure exerted by both the particles and the fluid, while the rear experiences only fluid forces. Thus, the net deflection of the diaphragm reflects the contribution of the particle forces only. That probe has been used to make the particie pressure measurements on the vertical side walls of gas-fluidized beds that were presented in Campbell \& Wang (1991) (although it could be used in many othur flow situations).

Campbell \& Wang (1991) showed that the particle pressures in gas-fluidized beds were largely generated by the passage of bubbles. In particular, they showed that the average particle pressure exerted on the side walls scaled with the average size of the bubble. This immediately brings to mind two questions: (1) what is it about bubbles that leads to particle pressure generations and (2) would there be measurable particle 
pressures in liquid-fluidized beds which, while unstable, do not bubble? This project is largely airned at answering these two questions.

The current project is designed to extend the work of Campbell \& Wang (1991) to further clarify the multiphase flow mechanics that lead to particle pressure generation. The first step was to improve the particle pressure transducer which has resulted in a publication, Campbell \& Rahman (1992). The first section of the report describes that work and is largely a reprise of the paper. The following two sections describe preliminary measurements in gas and liquid fluidized beds. The last describes some computer simulation studies of the flow of granular material in hoppers that is being partially supported using supercomputer time granted as along with this project. 


\subsection{IMPROVED PARTICLE PRESSURE TRANSOUCER}

Campbell \& Wang (1990) showed that there was room for improvement in the dynamic response of that probe to fast changes in the local fluid and/or particle pressures. The problems arose largely because, fluidd dynamically, the probe acted as the equivalent of an $\mathrm{RC}$ circuit in the way that it equulizes tho fluid pressure across the diaphragm. Ideally, the fluid pressure behind the diaphragm should exactly match the fluia pressure outside. Practically, equalizing the pressures requires thit a small quantity of fluid be passed from the test region at the front of the diaphragm, through the small passages, to the cavity at the rear of the diaphragm. The volume of the cavity determines the quantity of gas which must be moved and is the source of the apparent capacitance of the system. The resistance is applied by the small channels through which the fluid must move between the cavity and the outside. Now, an $R C$ circuit has two effects on the signal. Overall, it acts as a low pass filter; for high frequency pressure signals, the pressure behind the diaphragm simply does not have time to equalize with the applied pressure. As a result, the probe may show some response to rapid changes in air pressure, even in the absence of particles. But, even at lower frequencies, it will take some time for the pressures to equalize, resulting in a response that is phase shifted with respect to the applied signal. Campbell \& Wang (1990) used an audio speaker attached to a signal generator to apply an oscillating pressture signal to their probe and were able to demonstrate both of these features. Nonethel\%; a long time average of the signal will yield the average displacement of the diaphrigm so that neither of these problems affect the time averaged results presented by Campbell \& Wang (1991).

The probe described below virtually eliminates these problems by reducing both the resistance and capacitance of the system.

\subsection{NEW TRANSDUCER DESIGN}

A schematic of the improved transducer is shown schematically in Figure 2.1.1. Here, the solid diaphragm of the probe described by Campbell \& Wang (1990) is replaced by a screen. As the screen has orders of magnitude more area than the small holes that surrounded the diaphragm of the old probe, the resistance to fluid motion is dramatically reduced. At the same time, the capacitance of the probe is minimized by making the cavity behind the diaphragm as small as possible. (There is really no need 


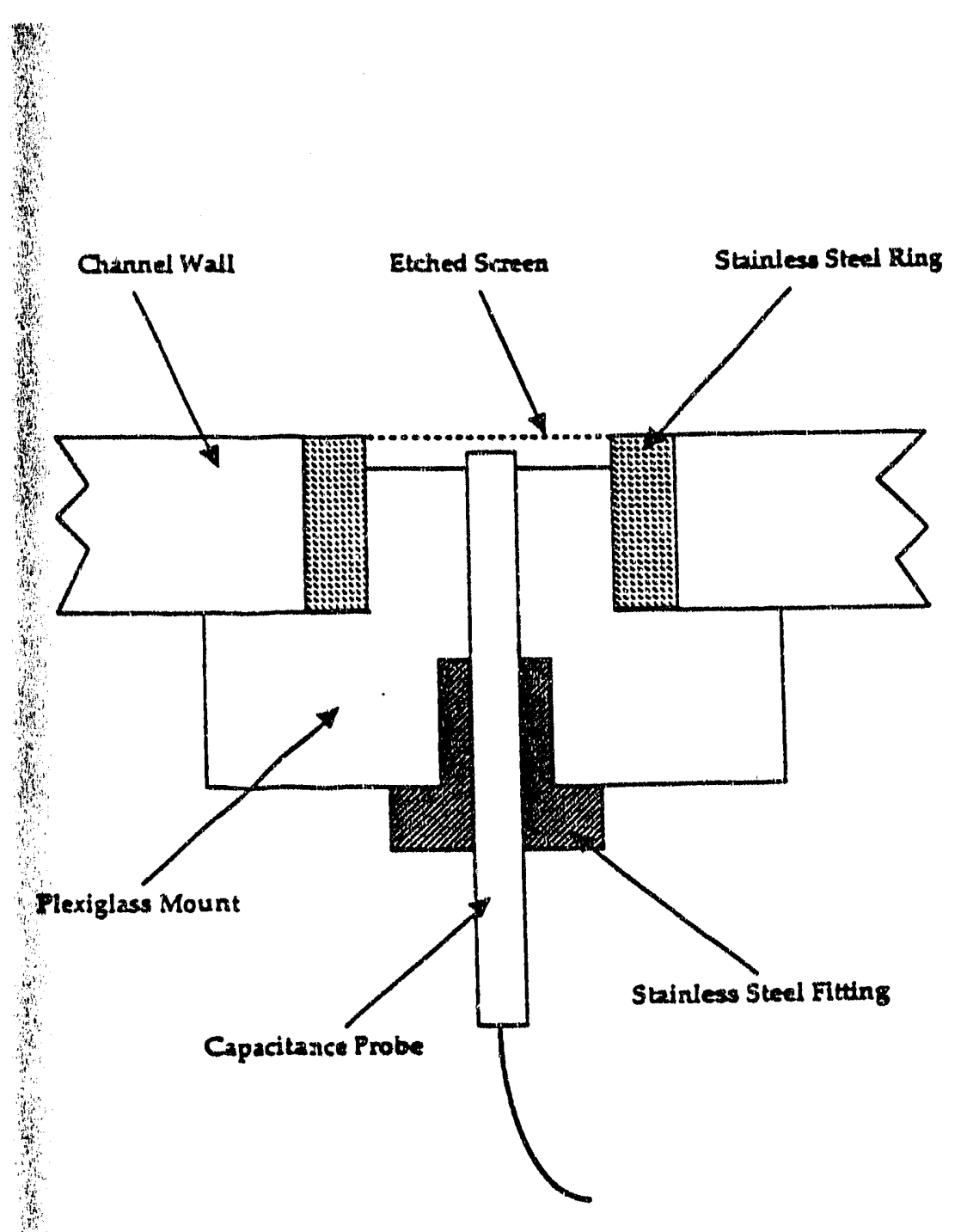

Figure 2.1.1: A schernatic of the new particle pressure transducer.

that the cavity be much thicker than the full range displacement of the diaphragm. In the current probe, the maximum measurable diaphragm motion is $0.13 \mathrm{~mm}$ ( $0.205 \mathrm{in}$.) and the cavity is cut to the same depth.)

There in however, another reason why the cavity should be kept as narrow as passible. If there is to be flow past the probe, thexe will generally be a fluid pressure gradient along the surface of the diaphragm. Such pressure gradients can be very large; for exaraple, in a fluidized bed, the pressure drop is approximately the weight per unit area of the section of particle bed, outside the probe. Under such conditions, the fluid might find it easier to pass through the screen, travel through the particle-sree cavity behind the diaphragm, and exit through the other side, rather than make the tortuous journey through the paxticle bed. This would alter the fluid flow pattern in the immediate vicinity of the screen and may bias the particle pressure measurement. Just such a problem was noted with the old probe. Originally, the solid diaphragm was 
surrounded by a ring of fluid passage ports that permitted a flow behind the diaphragm, but, eventually, all the holes were covered except for two located on diametrically opposite sides. The probe was tinen placed in the wall so that the diameter connecting the two ports was perpendicular to the external pressure gradient. Thus, there could be no mean pressure difference between the ports and no net flow behind the diaphragm. This change had a demonstrable effect on the measured pressures. In the original configuration, a maximum was observed in the average particle pressure at moderate fluidizing gas velocities which disappeared once all but the two fluid passage ports had been blocked. Making the cavity behind the diaphragm as narrow as possible, not only minimizes the capacitance, it maximizes the pressure drop that a fluid will experience when passing behind the diaphragm, making such a passage less attractive to the fluid. This appears to work as the particle pressure maximum, referred to above, is absent from the measurements taken with the new probe. Further improvements might be had by reducing the diameter of the diaphragm as much as possible so as to minimize the fluid pressure difference across the probe.

The construction of the probe should be quite evident from Figure 2.1.1. The screen that forms the diaphragm is tensioned and attached to a stainless steel ring by a spotwelding. We have built several versions of the probe with various diaphragm diameters; the one used in the tests presented at the end of this paper had a $12.7 \mathrm{~mm}\left(\frac{1}{2}\right.$ inch) diameter. The displacement of the diaphragrn is measured by a MTI Accumeasure probe with a $0-0.13 \mathrm{~mm}$ range that determines the displacement of the diaphragm by sensing the capacitance across the air gap between the probe and the diaphragra. Of course, the displacement could also be measured by mounting strain gauges or any other means, but this seemed to be the easiest scheme for us to implement.

The composition of the screen turned out to be very important to the success of this design. The first attempt to build this probe used a standard woven wire screen. But we found that spotwelding the screen to the ring broke many of the wires and the diaphragm could not retain sufficient tension. We then tried various designs that tensioned the screen like a banjo or drum head. There, the screen was attached to a outer metal ring which held the screen tightly over an inner "tone ring." Screws attached to the outer ring allowed the tension of the screen to be varied in much the same way as a drumhead is tuned. This design was also discarded as it showed a small nonlinear response, which, we were able to attribute to slipping of the screen over the tone ring. Eventually we abandoned the idea of using a woven screen altogether. 
Instead, we discovered that one could obtain stainless steel sheets, into which an extremely uniform pattern of circular holes had been etched by a manner akin to the manufacture of printed circuit boards. The screen we used in the current probe is manufactured by Buckbee-Mears Precision Etched Products Group (model \#2-2-8). The holes are approximately $70 \mu \mathrm{m}$ in diameter and are arranged in a bexagonal pattern on a $.05 \mathrm{~mm}$ (.002in) thick sheet. Because the screen is etched from a continuous sheet, spotwelding cannot cause enough structural damage (such as breaking the wires that make up a woven screen) to mair the screen unable to sustain tension. This allowed us to return to our original, and extremeiy simple, design which was shown in Figure 2.1.1.

Problems might arise in using this design in situations that contain fines that are small enough to pass through the screen that serves as the diaphragm. If enough particles leak through, they can fill the gap behind the diaphragm and restrict its motion. This could be a large problem for test materials that either have a wide size distribution or have been subject to sufficiently violent handling to cause a significant amount of particle attrition. In such a case, the probe would have to be disassembled and cleaned unless some other provision is made (such as a port allowing the gap to be vacuumed clean). How often such cleaning is required depends on the material type and the flow conditions.

Dynamically, the probe behaves as a simple diaphragm responding to the pressure difference between the interior and exterior. If both pressures are applied by the fluid only, the two should balance and there should, ideally, be no response. However, as mentioned previously, it will take some time for the pressure behind the diaphragm to balance that outside; the probe is the hydrodynamic equivalent of an RC circuit, where the resistance is the pressure drop needed to force the required flow across the screen and the capacitance is related to the volume of the cavity behind the screen, which determines the amount of fluid which must be moved. Any dynamically interesting

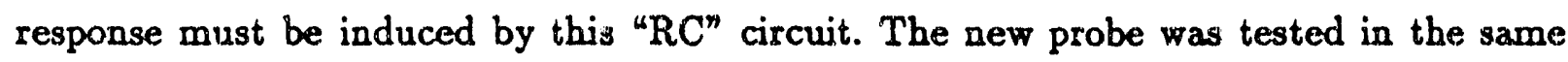
audio speaker apparatus used by Campbell and Wang (1990), which applies an oscillating air pressure to the probe. However, with the new design, the matching of the fluid pressures across the diaphragm occurs so quickly, that we were unable to detect any response of the probe to the applied pressure signal at any frequency tested (up to $1000 \mathrm{~Hz}$ ). We conclude that this new design has eliminated the resistance and capacitance problems associated with the old probe. 


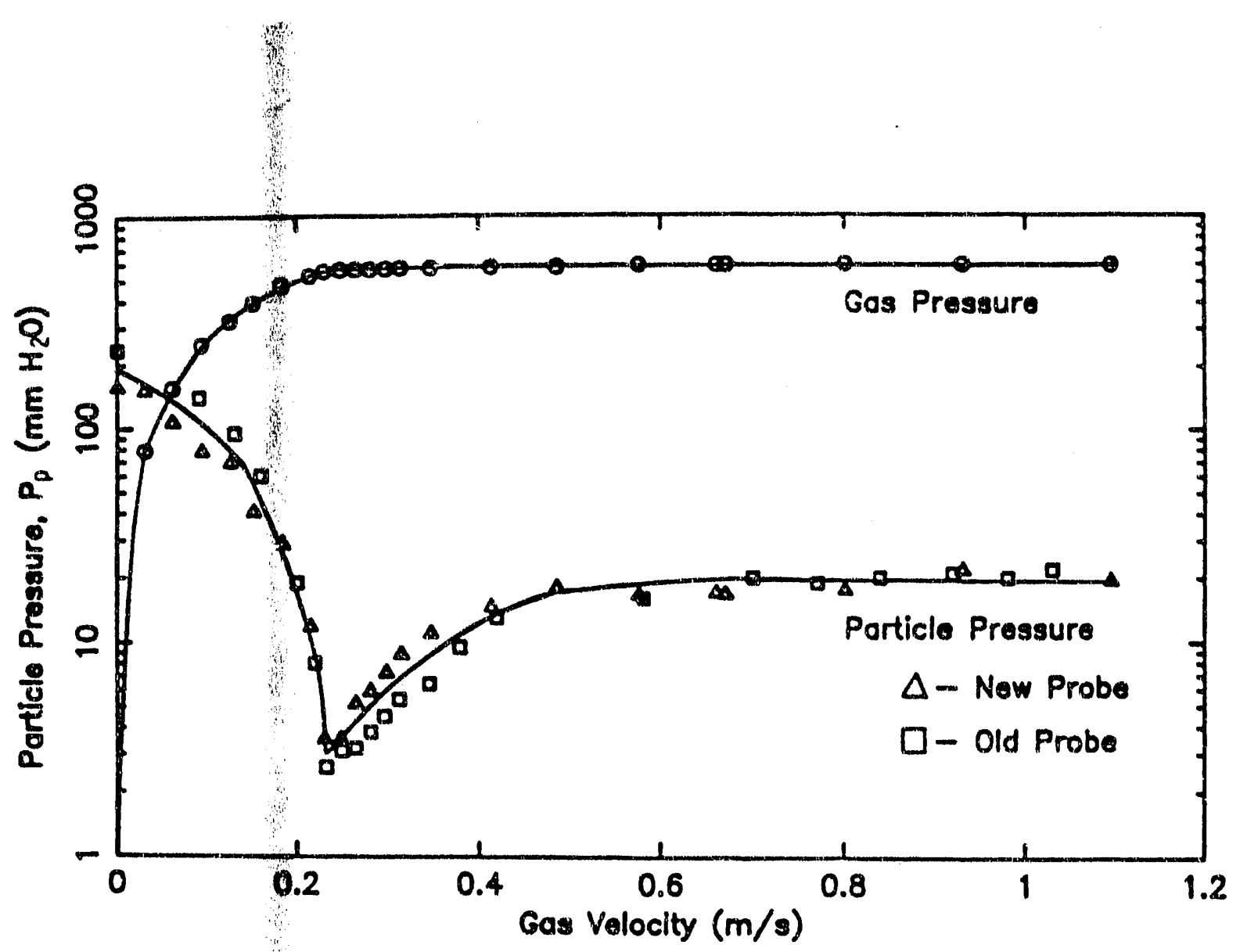

Figure 2.2.1: Comparison between the measurements of the old and improved probes. The results represent the average particle pressure measurements exerted on the sidewalls of a gas-fluidized bed. These data were taken in a $12.7 \times 12.7 \mathrm{~cm}$ square bed of $0.5 \mathrm{~m} m \mathrm{~m}$ glassbeads.

\subsection{ARCEETYPIOAT PARTICLE PRESSURE MEASUREMENTS}

Figure 2.2.1 shows a typical plot of time-averaged particle pressure measured in the side wall of a gas-fuidized bed. The measurements with the new probe are compared against nearly identical measurements made with the old probe. The plot also shows the gas pressure drop between the top and bottom of the bed. These particular results were taken with the probp positioned 15.2cm from the distributor for a test bed composed of $0.5 \mathrm{~mm}$ glassbeads. The gas pressure points show the characteristic of most such measurements. Thal is, the gas pressure difference initially rises rapidly from zero until it reaches a value equal to the weight of the bed per wnit area. At this point, the gas pressure difference is large enough to support the weight of the bed and the bed is said to be "fluidized." (Up to this point the bed is said to be "packed.") Further increases in velocity no longer change the pressure drop. The gas pressure curve gives a useful point of reference from which to understand the behavior of the particle pressure curve. (The 
reader might note that the behavior of the particle pressure is totally unlike that of the gas pressure.)

With zero fluidizing gas velocity, the particle pressure is large as the entire bed is supported only across interparticle contact points. Turning on the gas flow causes a drop in particle pressure (and a corresponding increase in gas pressure) as progressively more and more of the bed is supported by fluid forces. The particle pressure reaches a minimum, at just about the velocity where the bed is fully supported by gas pressure. Almost immediately after this point, the bed begins to bubble and the particle pressure begins to rise, presumably due to the agitation of the bed; beyond that point, the particle pressure seems to asymptote to a constant value when the bed is slugging. The results of Campbell \& Wang (1991) show that, in the fluidized region, the particle pressure scales with the mean bubble size within the bed and that the asymptote to a constant value for large fluidizing gas velocities is explained as the size of the bubbles becomes limited by the dimensions of the bed.

Figure 2.2.1 compares measurements taken with the new probe to similar measurements taken with the old. As can be seen, the two measurements are nearly identical. The major deviation between the two measurements occurs when the bed is packed. The results of Campbell \& Wang (1991) showed that the particle pressures measured in the packed bed region were dependent on the structure of the bed and that changing the structure (e.g. by tapping the walls and forcing the bed to settle) strongly altered the particle pressure measurement. As both measurements, shown in Figure 2.2.1, could not be taken at exactly the same time, we could not guarantee the same structure in the packed bed and, consequently, exact agreement of the results in the packed bed region should not be expected. The important observation to be made from this figure is that the two measurements are nearly identical in the fluidized region.

But remember that the purpose of this new probe was to allow better resolution of temporal events, not to make time averaged measurements. When the bed is fluidized, Campbell \& Wang (1991) found that the majority of the particle pressure is attributable to the passage of bubbles. Consequently, the actual particle pressure cannot be uniform, but, instead, must be applied in an unsteady manner as the bubble crosses the surface of the probe. With this in rnind, Figure 2.2.2 shows measurements of the instantaneous particle pressures measured on the sidewalls of the bed. Figure 2.2.2a shows that the particle pressures measured just beyond minimum fluidization are nearly 
uniform with time. However, the unsteady manner in which the particle pressure is applied is very anparent when the bed is freely bubbling (Figure 2.2.2b). Finally, when the bed is slugging, it can be seen that the particle pressure is applied in tall sharp peaks which are of, surprisingly, short duration (of the order of several milliseconds). 


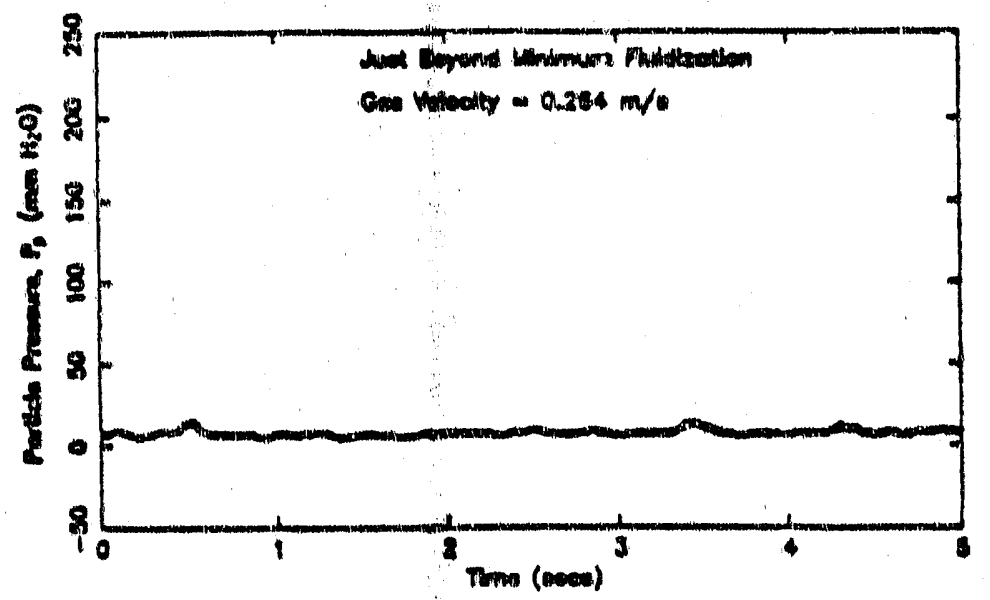

(a)

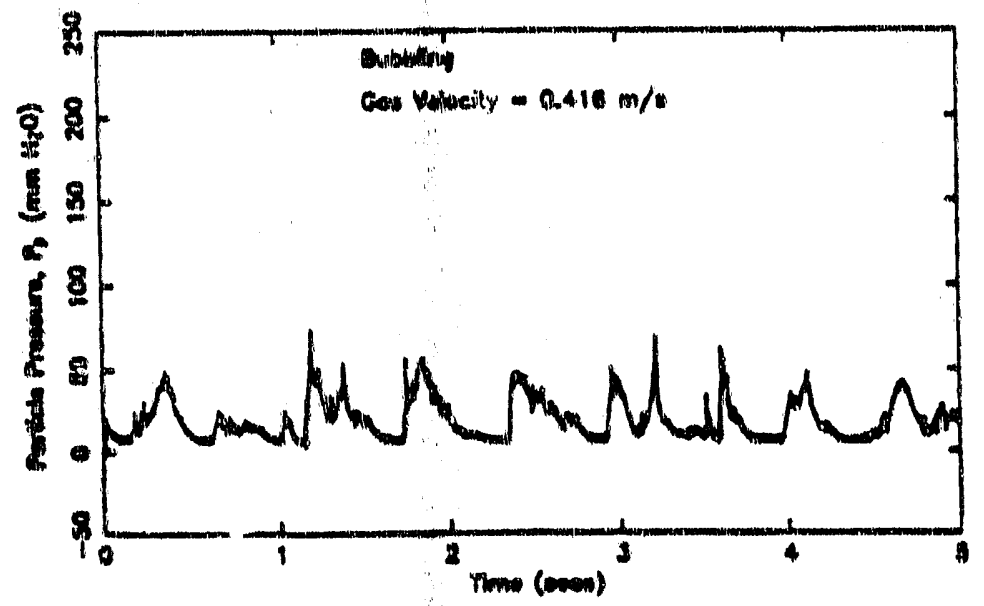

(b)

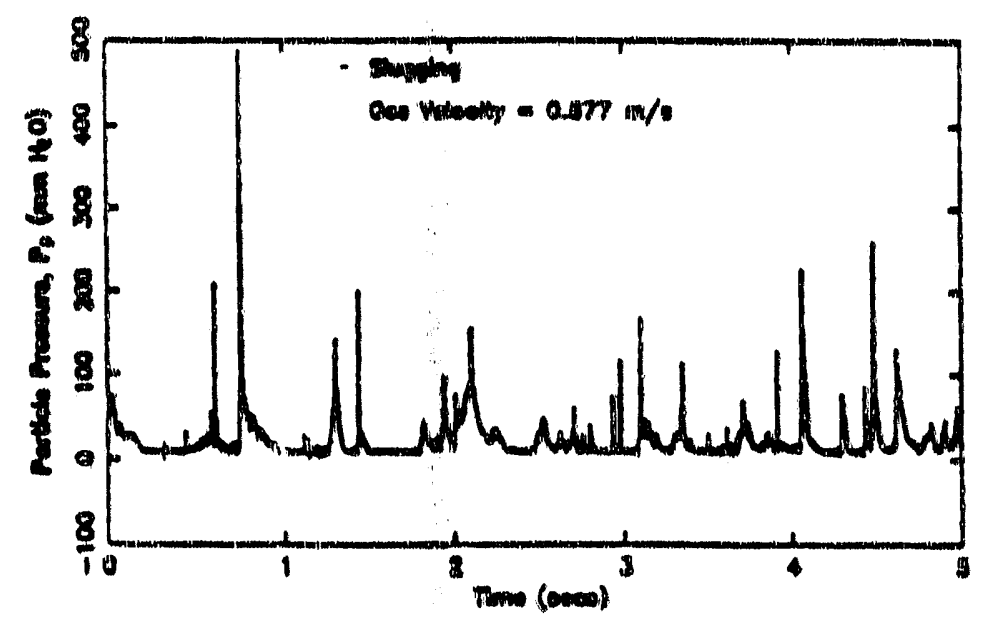

(c)

Figure 2.2.2: Instantaneous mearurements of the particle pressure exerted on the side wall of the gas gludized bed that were taken with the new probe. (a) just beyond miniman Iluidization, (b) a steadily bubbling bed, (c) a slugging bed. 


\subsection{PAR'TICLE PRESSURE MEASUREMENTS AROUND SINGLE BUBBLES IN A TWO-DIMENSIONAL GAS-FLUIDIZED BED}

The results of Campbell and Wang (1991) indicate that particle pressures, measured along the side wall of a gas-fluidized bed, are primarily generated by the passage of bubbles. The primary evidence lies in the observation that the average values of the particle pressure scales with the equivalent diameter of bubbles. However, the pressure obviously cannot be uniform across the bed. In particular, the particle pressure must go to zero in the particle-free region inside the bubble. Furthermore, the side wall of the bed may be a peculiar region as the particles pushed aside by the passage of a bubble cannot cross the wall, and therefore, the walls must affect the bubble motion in their immediate neighborbood.

The easiest way to determine the distribution of the particles pressure around a bubble is to perform the experiment in a "two-dimensional" fluidized bed. This is a tarm used to describe beds that are extremely thin in one dimension. In such a situation, bubbles span the small breadth of the bed so that it is possible to make a measurement of the particle pressure across a bubble without actually inserting a probe in the bed. The position of bubbles may be localized by artificially injecting bubblis into a bed held near minimum fluidization.

Figune 3.0.1 is a schematic of the two-dimensional bed we have built in the last year. The test section is $60^{n}$ high, $18^{n}$ wide, but only $1^{n}$ deep. It is fed by an air supply system that passes air through a ten inch packed bed and two of the Buckbee-Mears (22.8) $70 \mu \mathrm{m}$ etched screens to assure a uniform airflow. The air flow is set so that the bed is at minimum fluidization conditions. Then bubbles may be injected through a porous plate covered port, located 9 inches above the distributor. The bubble injector consists of a series of plenums which are pressurized through a precision pressure regulator. Firing a solenoid valve admits the additional air to discharge into the bed, causing a bubble to form. Different plenum pressures will discharge different quantities of gas. This system allows the injection of bubbles of repeatable size. Finally 16 ports are cut into the face of the channel to admit the particle pressure transducers. The ports are configured in 4 lines of 4 , spaced at 6 inch intervals above the injection port. In each line, the ports are spaced 2 inche apart, spanning the area from the center of the bed to one wall. Four particle pressure probes, of the type described in the last section have been built and may be inserted in any of the ports. 


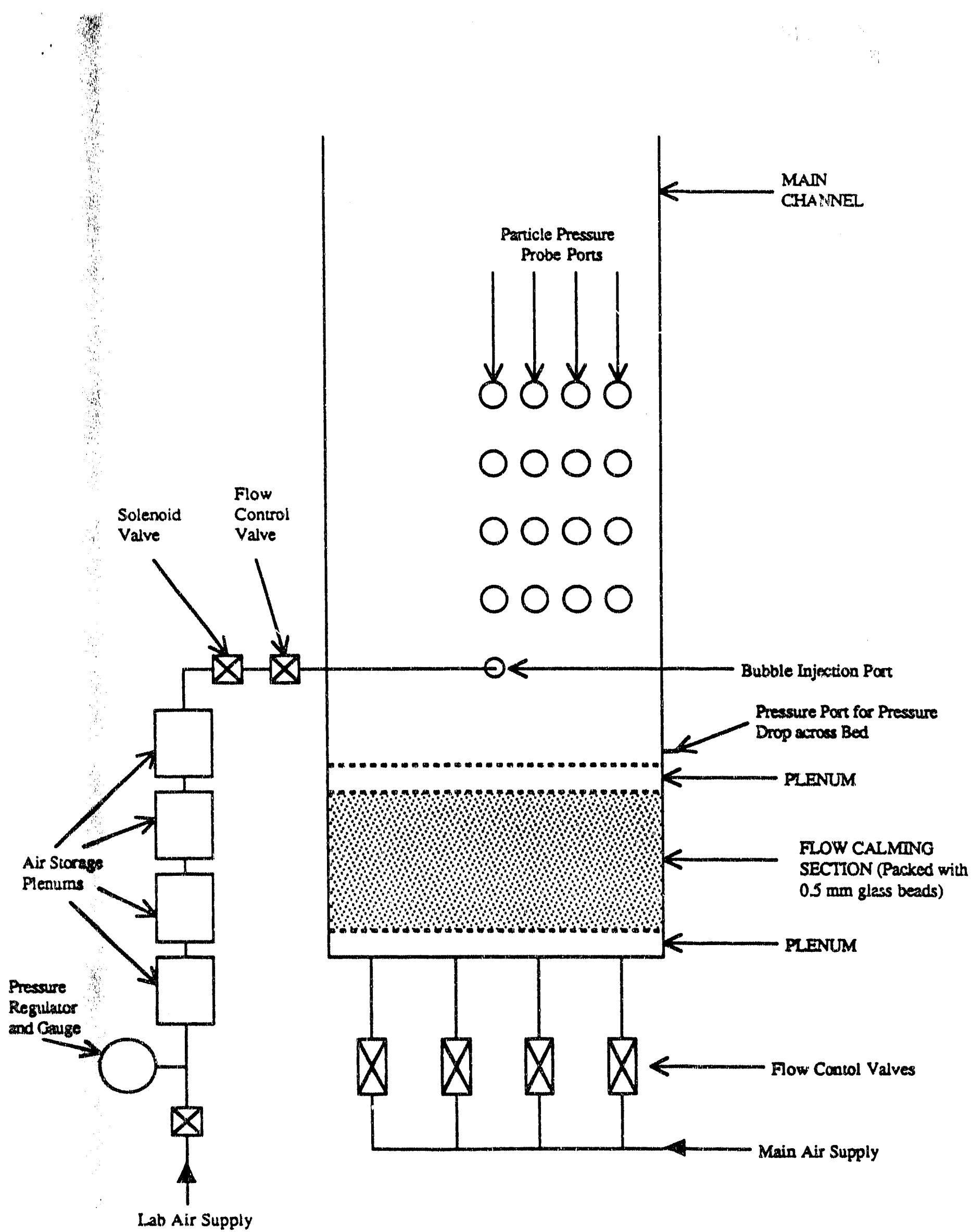

Figmere 3.0.1: Schematic of the two-dimensional tuidized bed. 
Eventually, we plan to use an image processing system to locate the bubble relative to the probes and to evaluate its size. With time traces of the particle pressure and precise knowledge of the bubble location and size, it will be possible to trace the distribution of particle pressure about the bubble. (However, as the bubble size will increase as it rises, there is an implicit assumption in this description that the particle pressure distribution scales with the bubble size. This can easily be checked by making the measurement around bubbles of several sizes, but, as Campbell \& Wang (1991) show that the average particle pressures scale with bubble size, it would be surprising if the same were not true for the instantaneous measurements.) We have held off purchasing the equipment as significantly improved products are becomin and migit as well be waited for, as, with all of the construction, we bave had no time to develop the image processing software. We have been waiting for the introduction of the a Data Translation 3851 frame grabber that is available with 8MB of memory, sufficient to hold 1 second of acquired images, before dumping to disk. This will allow a high spatial resolution of the bubble location and size. A software package available from the same source, Global Lab Image contains routines that evaluate the center of gravity and area of the bubble - exactly the information neecled. J.he board has actually been available for a few months, however, the support software will not be available until September. In the meantime, a new board that performs similar functions, with software available, has recently become available from Imaging Technologies and we are awaiting further information before making a final decision.

However, it is possible to obtain the approximate position of a bubble from the local change in the gas pressure. Such changes have been predicted by many analysts. In particular, one of the earliest studies, Davidson (1961), has also proven to be one of the most successful in predicting the gas-pressure distribution. Littman \& Holmolka (1970) have used the unique gas pressure signature as a way of locating bubbles and determining their rise velocities. Now, the gas pressure changes caused by the passage of a bubble are small, and we do not currently have any pressure transducers that can accurately resolve the pressure. However, the measurements we can make are sufficient to localize the bubble.

Figure 3.0.2 shows preliminary particle pressure measurements performed on the two-dimensional bed filled with $0.5 \mathrm{~mm}$ glassbeads. All four measurements are taken from probes mounted in the ports located 18 inches above the bubble injector. Each plot 

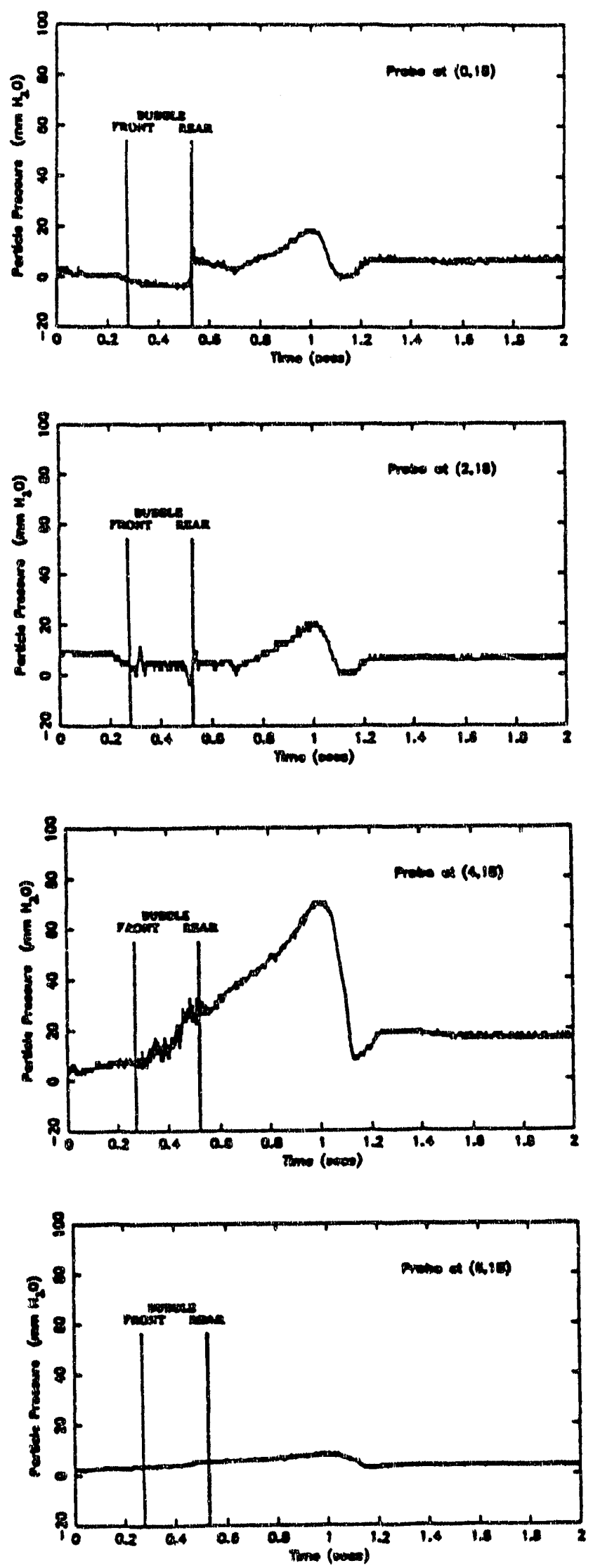

Figure 3.0.2: Time histories of the particle pressure surrounding a bubble rising in a two-dimensional gas fluidized bed. All of this data was taken with the probe mounted. eighteen inches above the bubble injection port. 
is marked with an ordered pair of the form $(n, 18)$ where $n$ is the distance from the bed centerline and 18 represents the height above the bubble injector (i.e. $(0,18)$ is the probe mounted in the center of the bed, $(2,18)$ is the probe mounted 2 inches off the centerline, and so on). An eyeball estimate gives the size of the bubble as 5-6 inches; thus the $(0,18)$ probe passes through the bed center, the $(2,18)$ probe may only become uncovered during the middle of the bubble passage, while the other two probes, $(4,18)$ and $(6,18)$ are completely immersed in the bed throughout the experiment. The particle pressures are plotted as a function of time and are marked with lines that show the front and the rear of the bubble (as determined from the gas-pressure distribution). The most interesting part of this figure is that the particle pressures are largely generated in the particle wake. Particle pressures upstream of the bubble are small (this would be expected from the results of Campbell \& Wang (1991) which show that the particle pressures are insignificant around minimum fluidization). This is consistent with forces measured on tubes that were presented by Professor Roland Clift at the IUTAM Symposium on the Mechanics of Fluidized Beds, held at Stanford last summer, which also so that the largest stresses appear in the wake. More interesting though, is that the largest magnitudes of the particle pressure are measured by the $(4,18)$ probe which is mounted just outside the region through which the bubble passes, although it dies off quickly by the time it has reached the $(6,18)$ probe. These are very interesting results and will be verified and studied in much greater detail. 


\subsection{PARTICLE PRESSURE MEASUREMENTS IN WATER FLUIDIZED BEDS}

Similar measurements may be made in a bed which is fluidized by water rather than gas. Unlike gas fluidized beds, liquid fluidized beds do not bubble, even though they are unstable and exhibit density waves that have often been speculated, though never observed, to grow into bubbles. While, there are limited industrial applications of liquid fluidized beds, they have been widely studied as a way of understanding the stability of the beds. As such, the particle pressures measured therein are of particular importance as the particle pressure is the most frequently employed mechanism to explain the apparent stability (see, for example, Batchelor (1988), Garg \& Pritchet (1975) and Mutsers \& Rietema (1977)). In general, such studies indicate that if the particle mass behave somewhat elastic: lly, i.e. if the particle pressure increases as the void fraction is reduced and thus puts up resistance to void fraction changes, the bed might be stable. As liquid beds are more stable than their gas-fluidized counterparts, the particle-pressure measurements may demonstrate any such elastic behavior and thus help understand whether any such mechanism is active.

The liquid fluidized bed is shown schematically in Figure 4.1.1. The system will be fed water by a system of three positive displacement gear pumps that may be used separately or collectively to allow fine control of the superficial velocity of the fluid. To control the flow rate, the speed of the pump drive motors is controlled by an AC variable frequency inverter. The flowrate is monitored by a vortex flowmeter and, again, the flow may be diverted through rotameters, to monitor the small flowrates for which the vortex flowmeter does not operate accurately. To provide a uniform velocity field inside the bed, the flow is passed through a 10 inch long packed particle bed composed of $0.5 \mathrm{~mm}$ glassbeads which is topped by several layers of fine screens. The same etched screens, Buckbee-Mears Precision Etched Products Group (model \#2-2-8), that are used for the diaphragm of the particle pressure probe, are used here. After passing through the fluidized bed, the water is passed through a second short packed bed, composed of $1 \mathrm{~mm}$ glassbeads, before being collected at a plenum at the top of the channel and returned through the pump. This second packed bed is used to eliminate any upstream disturbances in the flow that might be induced by the plenum. Eight valves guide the path of the water flow through the control system. By the proper setting of the valves, the pumps may be used to circulate water through the bed in the standard manner, fill the bed, empty the bed, purge trapped air, or direct the flow through any of the three flowmeters. 


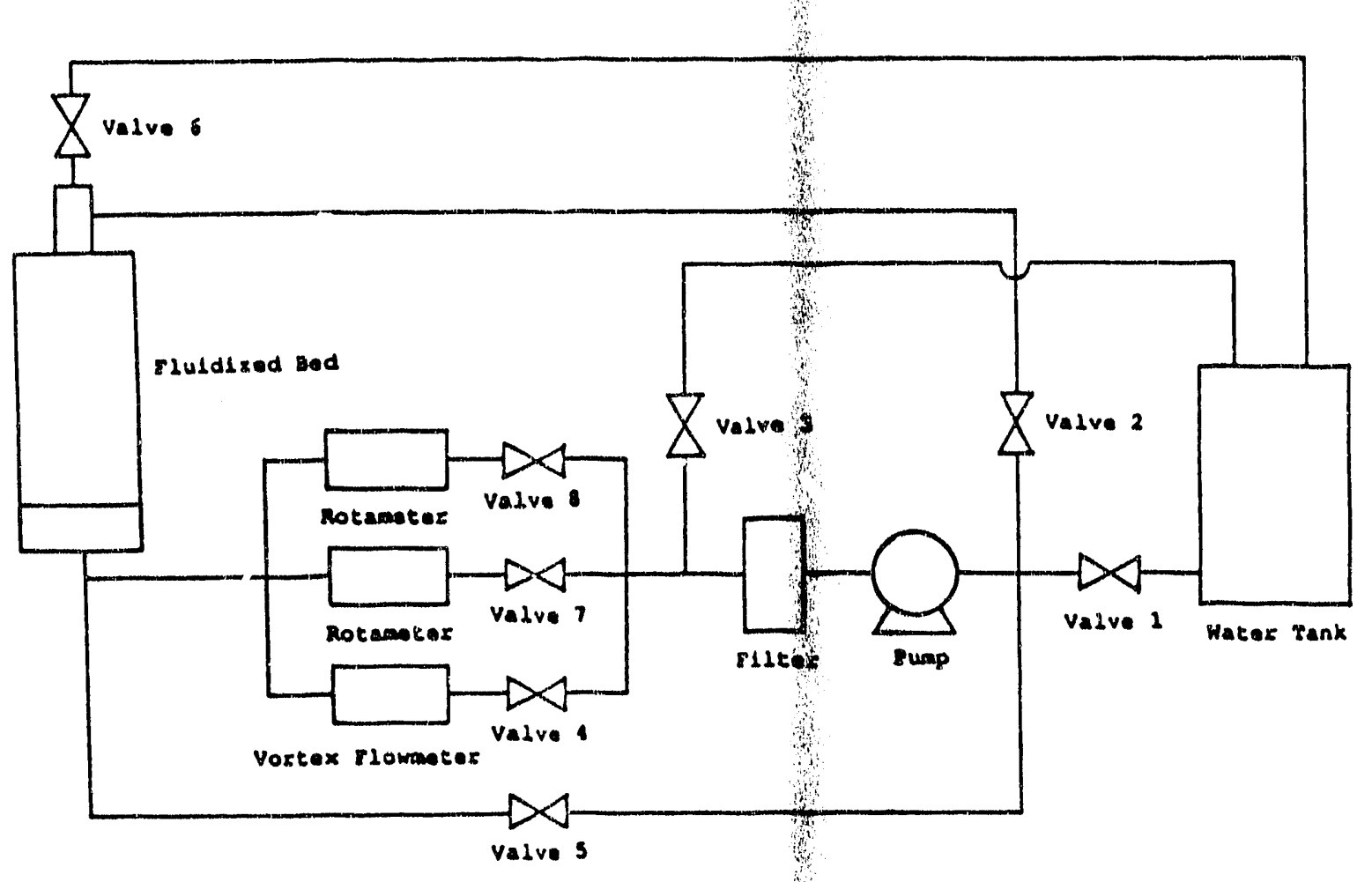

Figure 4.0.1: Schematic of the liquid fluidized bed

The particle pressure probe used in these expeximents will be essentially the same as that shown in Figure 2.1.1. The major difference is that the gap behind the diaphragm will be filled with water instead of air.pue to the relatively small resistance of the water, the capacitance probe used to measure the displacement of the diaphragm cannot be used in the liquid fluidized bed. Our fint attempt to solve this problem was to use the resistance of the water between an electrode (located at roughly the same position of the capacitance probe shown in Figure 2.1.1) and the diaphragm to make the same measurement. The electrode/diaphragm citcuit formed one leg of a resistance bridge. The bridge was excited with a high freftency AC signal from a Daytronics strain gauge signal conditioner; the AC signal prevented the water molecules from becoming polarized and biasing the measurement, It was understood, when this project was started, that this would be a difficult measurement as changes in the conductivity of the test fluid, due to temperature changes or the presence of impurities, would alter the measurement. This requires that the conductivity of the fluid be monitored during the measurement - an extremely awkward proces. Even so, it was difficult to get a repeatable calibration.

Recently, we became aware of a new kind of displacement transducer that monitors 


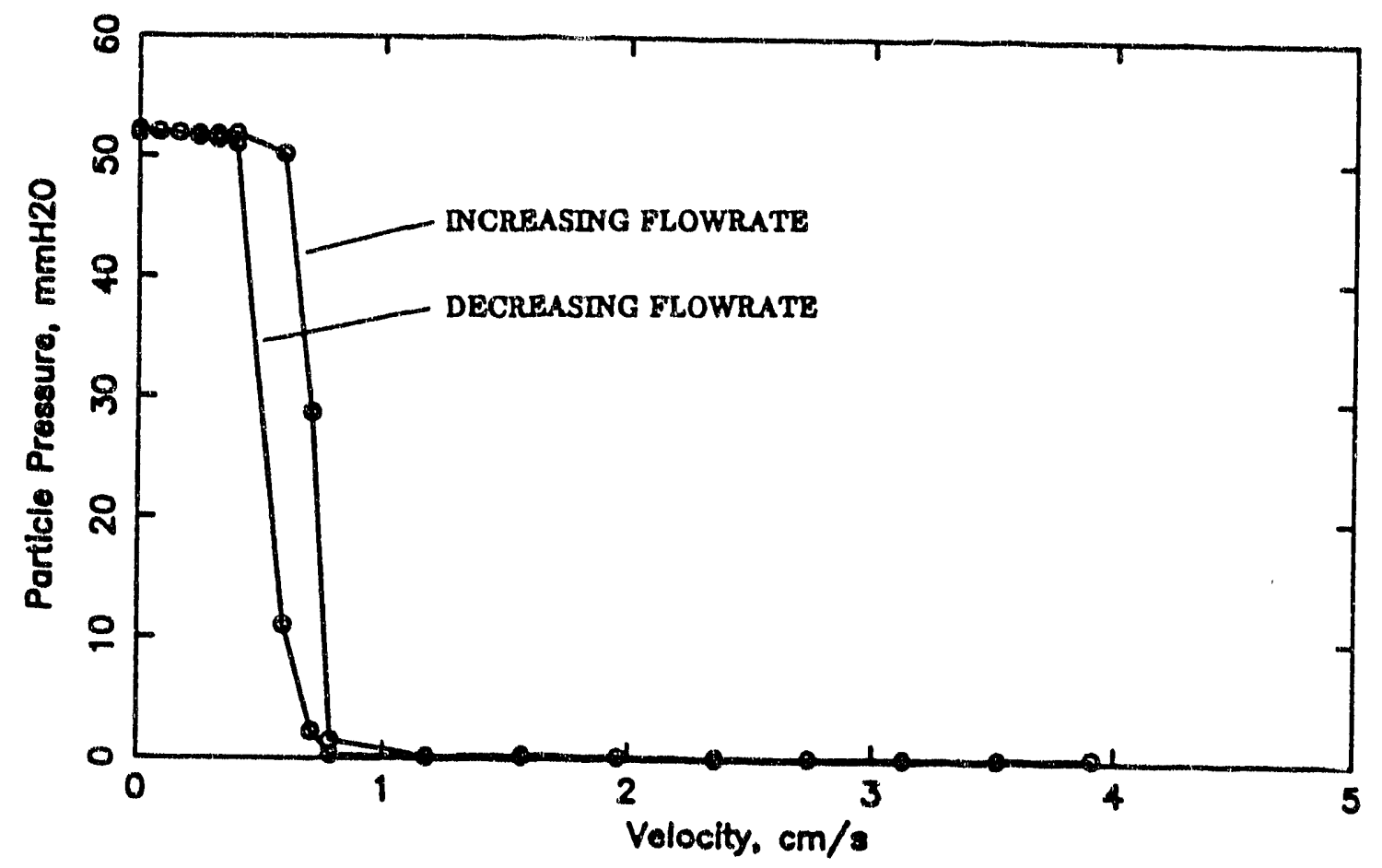

Figure 4.0.2: Preliminary particle pressure measurements made on 1.0mm glassbeads in a water fluidized bed

the eddy currents generated in ferrous materials. As the eddy currents are relatively independent of the conductivity of the surrounding fluid, this device can be used in a wate: environment. The sensor is manufactured by Keyence Cirp. We are using a AH305 sensor head and a AS-440-01 controller.

We are still evaluating the use of this sensor and the proper construction of a probe to make measurements in liquid fluidized beds. Difficulties arise from the extremeiy small values of the particle pressures generated in liquid fluidized beds, which, unfortunately, were unknown at the time that we started this work. The sensitivity of the particle pressure probe depends on the area of the diaphragm and the degree to which the diaphragm is tensioned. The current version of the sensor has a 1 inch diameter diaphragm and the diaphragm is as loose as we feel to be prudert. (The tension of the diaphragm cannot be indefinitely relaxed as it is the tension that applies the restoring force that resists the particle pressure.) It has a resolution of about $2.0 \mathrm{mmaH}_{2} \mathrm{O}$.

We have tested this probe on both $0.5 \mathrm{~mm}$ and $1.0 \mathrm{~mm}$ glassbeads in a water 
fluidized bed. Unforfunately, this is not sufficient to resolve the particle pressures generated when the bed is fluidized. Figure 4.0.2 shows the measured particle pressures exerted on the sidewall of a water-fluidized bed by a bed composed of $1.0 \mathrm{~mm}$ glassbeads. Reasonably large pressures are exerted when the bed is packed. These drop dramatically to zero (the actual numbers are somewhat smaller than 1.0rnm of water, well below the resolution limit of the probe) as soon as the bed becomes fluidized. No noticeable change can be observed thereafter. While all this is going on, the particles in the bed are noticeably agitated so that particle pressures should be generated. (Also, the bed has expanded from a void fraction of about $0 . .44$ to a value of about 0.69 .) Note that the same kind of hysteresis between the particle pressures measured with increasing velocity and decreasing velocity when the bed is packed, as was observed in gas fluidized beds by Campbell \& Wang (1991).

As this report is oeing written, the probe is being modified to be more responsive to small pressures $\mathrm{by}$ increasing the diameter of the diaphragm. The ports in the current bed will admit a diaphragm of nearly 2 inches in diameter. This should be approximately four fimes as sensitive as the current probe. But the problem with using a larger diaphragm is that it increases the pressure difference between the top and bottom of the probe; this encourages the fluid to bypass the packed bed and travel through the gap behing the probe, possibly biasing the measurement. This problem can be partially avoided by realizing that the diaphragin will deform into a circular arc; thus the gap behind the diaphragm need not be of constant depth, but may follow the shape of the deforined diaphragm. Such an arc would have such a large radius of curvature thit it would be nearly impossible to machine. (As the gap is approximately 0.005 in deep, the radius of curvature for a spherical cap shaped gap, 1 inch across, would have to be approximately 100 in.) Instead the gap will be cut into the shape of concentric circular steps, which will yield approximately the same resistance to flow, but be much easier to machine.

The final aspeet of the liquid fluidized bed research will be to build a two dimensional bed similar to that used to make instability measurements by Didiwania \& Homsy (1981). As in that study, density waves may be measured and observed from the light attenuation through the bed. In this case, we hope to make density maps using the same image processing system that was described in the last section. The goal was to try and relate the particle pressures generated to the magnitude and speed of the instability. At this time we have evaluated the light attenuation mechanism and 
performed a rough calibration. We had been holding off on the final construction until the problems with the particle pressure probe had been resolved, but have changed ou: minds and are currently constructing the bed. This will allow us, in the meantime, to get some experience with the density measurements. 


\subsection{CCMPUTER SIMULATION OF HOPPER FLOW}

Soon after the proposal for this project was accepted, we received an unexpected offer for supercomputer tinfe at the NERSC center in Livermore. While there was no direct need for supercornputter time for the particle pressure project, there were many related problems that could benelit from the offer. In particular, the P.I. has a great deal of experience in computer simulation of granular flows. Recently, the capability in this area has grown to the point that large scale systems might be simulated. For example, the P.I. has simulated landslide-like events in two-dimensions that are comprised of up to $1,000,000$ particles.

One such problem that has long gone unexplained and unstudied is the unsteady nature of the flow and the formation of ruphure zones in two-dimensional hoppers that were observed by Blair Fish \& Bransby (1973) and Lee, Cowin \& Templeton (1974). In a plane hopper, the flow is not symmetric about the center but alternates, first flowing along on $\epsilon$ wall and then along the other. Furthermore, this unsteadiness is associated with periodic fluctuations of the stresses exerted along the sloping walls of the hopper. When examined by $\mathbf{x}$-ray, it was discovered that rupture zones of slightly reduced particle concentration, taking roughly the shape of circular arcs, form behind the flowing section at the corker between the bin and the hopper. (The concentration in these rupture zones was reduced enough so that they showed up in the $\mathrm{x}$-rays, but, as the zones moved with the hlow, was clearly still large enough that the particles were still in contact with one another.) These observations have largely remained unexplained curiosities since the publication of those two papers.

Such observations aro perfect targets for the technique of computer simulation. Computer simulations of granular flow are descendants of molecular dynamics calculations in which each particle within a mechanical system of particles is followed exactly as it moves and ifteracts with its neighbors. The simulations depend only on models for the individual particle properties and the ways in which the particles interact with each other and the boundaries of the system, and hence may be considered idealized experiments performed on idealized materials. Computer simulation has played a valuable role in the development of granular flow understanding because literally everything is known about the simulated systems. In contrast, experimental studies are hampered. by 4 general lack of instrumentation and experimental strategies for performing work in a dense, abrasive medium that does not take well to intrusive 

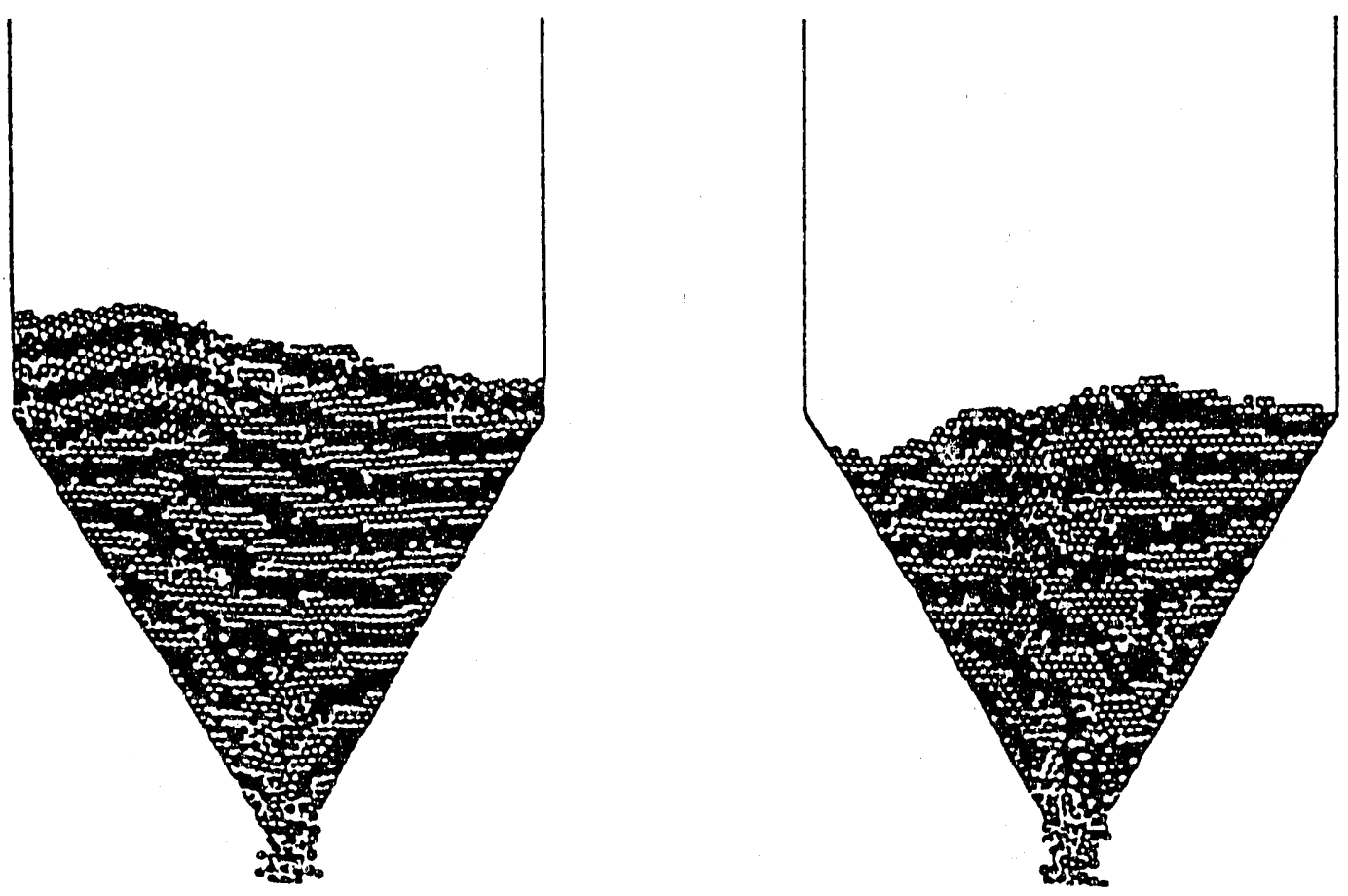

Figure 5.0.1: Two snapshots from a small scale test of the hopper flow simulation; the unsteady behavior, i.e. moving on one side and thein the other, can be seen from the shape of the free surface at the top and from the deformation of internal lines of "dyed" particles.

probing. Thus, r ost detailed measurements of density and temperature profiles and stress tensors cone from computer simulation rather than direct experiments. The computer simulation field has been reviewed by Campbell (1986) and Walton (1986).

The simulation technique used is the soft particle method for granular flows (see the :eview of simulation techniques by Campbell (1986)). Here, as in all discrete model computer sirnulations, the trajectory of every particle in followed exactly as it interacts with the other particles with which it comes into contact. Each interparticle contact is modeled as a spring and dashpot in the normal direction and a spring and surface friction in the tangential direction. The springs provide a restoring force to push the particle surfaces apart (which, naturally, disappears as $500 \mathrm{n}$ as the particles lose contact) and a combination of the dashpot and surface friction dissipate some of the kinetic energy of the particles and account for the collisional inelasticity. (It is casy to show that a constant dashpot coefficient is equivalent to a constant coefficient of restitution for a binary collision.) A soft particle sirnulation may be thought of as the simultaneous numerical integration of all the equations of motion that describe the 


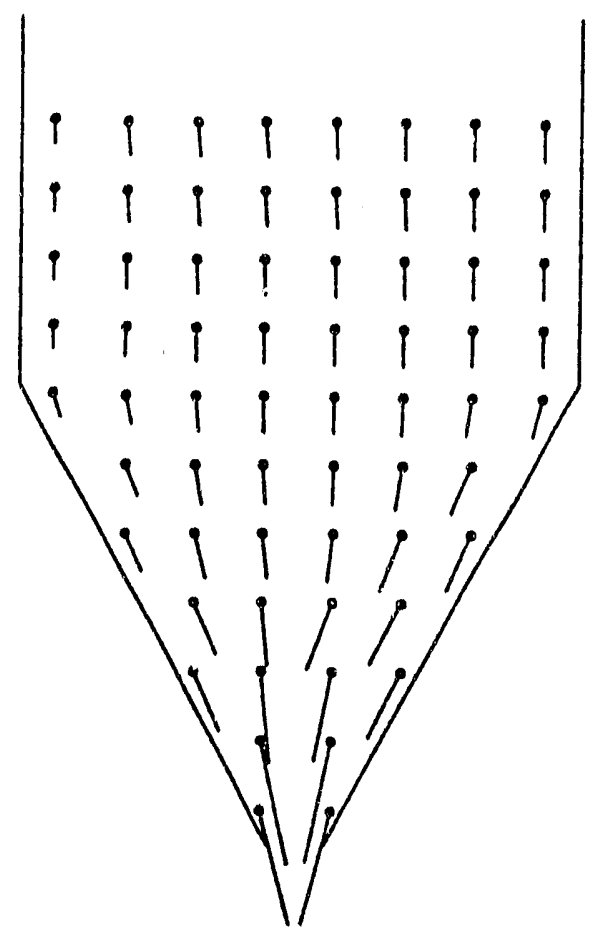

(a)

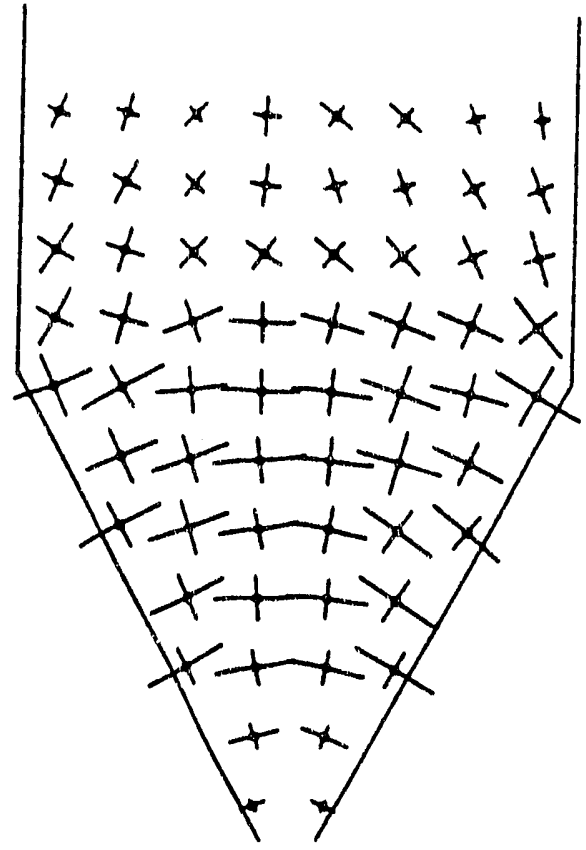

(b)

Figure 5.0.2: (a) The average velocity field inside the hopper. The length of the lines is proportional to the velocity and they direction of the local velocity. (b) The Average stress field. The lengths and orientations of the crosses reflect those of the principle stresses.

translation and rotation of all the particles in the system. For an equation of motion, the integration is made with respect to time and the time-step of the numerical integration is the time increment at which the simulation proceeds. Each particle responds to an omnipresent dowaward-pointing gravitational force as well as forces applied through contacts with other particles. The spring/dashpot used to model the contact requires typically 20-50 timesteps to accurately calculate each contact, requiring the simulation to proceed extremely slowly.

Some snapshots of the computer simulation are shown in Figure 5.0.1. The simulated situation consists of a vertical bin connected to a hoppex of $30^{\circ}$ (measured from the vertical) wall angle. This is a two-dimensional simulation and the particles are two-dimensional discs; this is done as, without having to fill out the third dimension, fewer particles are required to simulate larger systerns. These figures were taken frora a test performed on a desktop workstation, using approximately 7,000 particles. (We have just recently moved the problem to a Cray and are awaiting the first results.) The 
unsteady nature of the discharge can be observed from the deformation of the lines of "dyed" particles, but is more easily seen from the waviness of the free surface. Note, that no concentration reduction is noticeable in these figures. But, as was noted above, the actual reduction appartint in the $x$-rays, must be small indeed as the particles never lose contact; this would limit the actual concentration change to a few percent or less. (Neither Lee et al. (1974) or Blair-Fish \& Bransby (1973), calibrate their x-ray measurements to the concentration and, consequently, no quantitative evaluation of the change is available.)

But the great advantage of the computer simulation technique is that it permits the evaluation of properties that are generally inaccessible to ordinary experimental techniques. Two examples are shown in Figure 5.0.2. Figure 5.0.2a shows measurements of the average velocity of the particles. (These were taken from s simulation that differed from that shown in Figure 5.0.1, in that the particles are recycled through the control volurne in order to achieve a steady state situation in which the hopper does not ernpty. I.e. when a purticle passes through the hopper opening, we feed it back in the top of the hopyer.) The velocities are averaged over all the particles in small regions and are plotted as a single vector emerging from the center of the averaging volume, pointing in the direction of motion, with a length proportional to the average velocity.

Figure 5.0.2b shows the average stress distribution in the same hopper. Again, these stresses are averaged over small volumes. What we have plottied here are crosses that represent the principle stresses; the length of the lines represent the magnitude of the principle stresses and the orientation of the cross reflects the orientation of the principle stresses. Notice that the stresses go to zero near the exit. Far above the opening, one expects that the material is in static equilibrium; i.e. the flow is not: accelerating and the interpal stresses exsctly balance the gravitational forces. However, sornewhere near the opening, the stresses must go to zero leaving the particles in near frectall.

The main purpose of the averayce velocity and stress distribution is to provide a baseline with which instantaneous values - those that reflect the unsteady nature of the How - might be compared. We speculate that the appearance of rupture zones is related to an unsteady manner by which the internal stress state is relaxed from its static value to zero near the opening. 


\subsection{REFERENCES}

Batchelor, G.K., 1988, A new thenry for the instability of a uniform fluidized bed, Journal of Fluid Mechanics, 193 75-110

Blair-fish, P.M., and Bransby, P.I. 1973, Flow patterns and walls stresses in a rnassflow bunker, J. Eng. for Ind. 95, 17-26

Campbell, C.S. 1986, Computer simulation of rapid granular flows, Proc. $10^{\text {h }}$ US National Congress of Applied Mechanics, Austin Texas, June 1986

Carnpbell, C.S. and Wang, D.G., 1990, A particle pressure transducer suitable for use in gas-fluidized beds, Measurement Science and Technology, 1, 1275-1279

Campbell, C.S. and Wang, D.G., 1991, Particle pressures in gas-fluidized beds, Journal of Fluid Mechanics, 227, 495-508

Campbell, C.S., and Rahman, K., (1992) An improved particle pressure transducer, Measurement Science and Technology, 3 (no page number yet assigned)

Davidson, J.F., 1961, in Symposium on fluidisa tion - discussion, Trans. Instn. Chenn. Engrs., 39, 230-232

Didwania, A.K. and Homsy, G.M., 1981, Flo' regimes and flow transitions in liquid Đluidized beds, Int. J. Multiphase Flow, 7, 563-580

Garg, S.K. \& Pritchet, J., 1975, Dynamics of gas-fluidized beds, J. Appl. Phys. 46, 4493-4500

Lee, J., Cowin, S.C., and Templeton, J.S., 1974, An experimental study of the kinernatics of flow through hoppers, Trans. Soc. Rheology, 18:2, 247-269

Littman, H. and Homolka, G. A. J., 1970, Bubble rise velocities in two-dimensional gasfluidized beds from pressure measurements, Chem. Engng. Prog. Symp. Ser. No 105, 66, 37.46

Mutsers, S.M.P and Rietema, K., 1977, The effect of interparticle forces on the expansion of a homogeneous gas-fluidized bed, Powder Technology, 18 239-248

Walton, O.R., 1986, Discrete particle cornputer methods for modelling granular flow, Proceedings, ASCE meeting, June 3-6, 1986, Minneapolis Minn. 

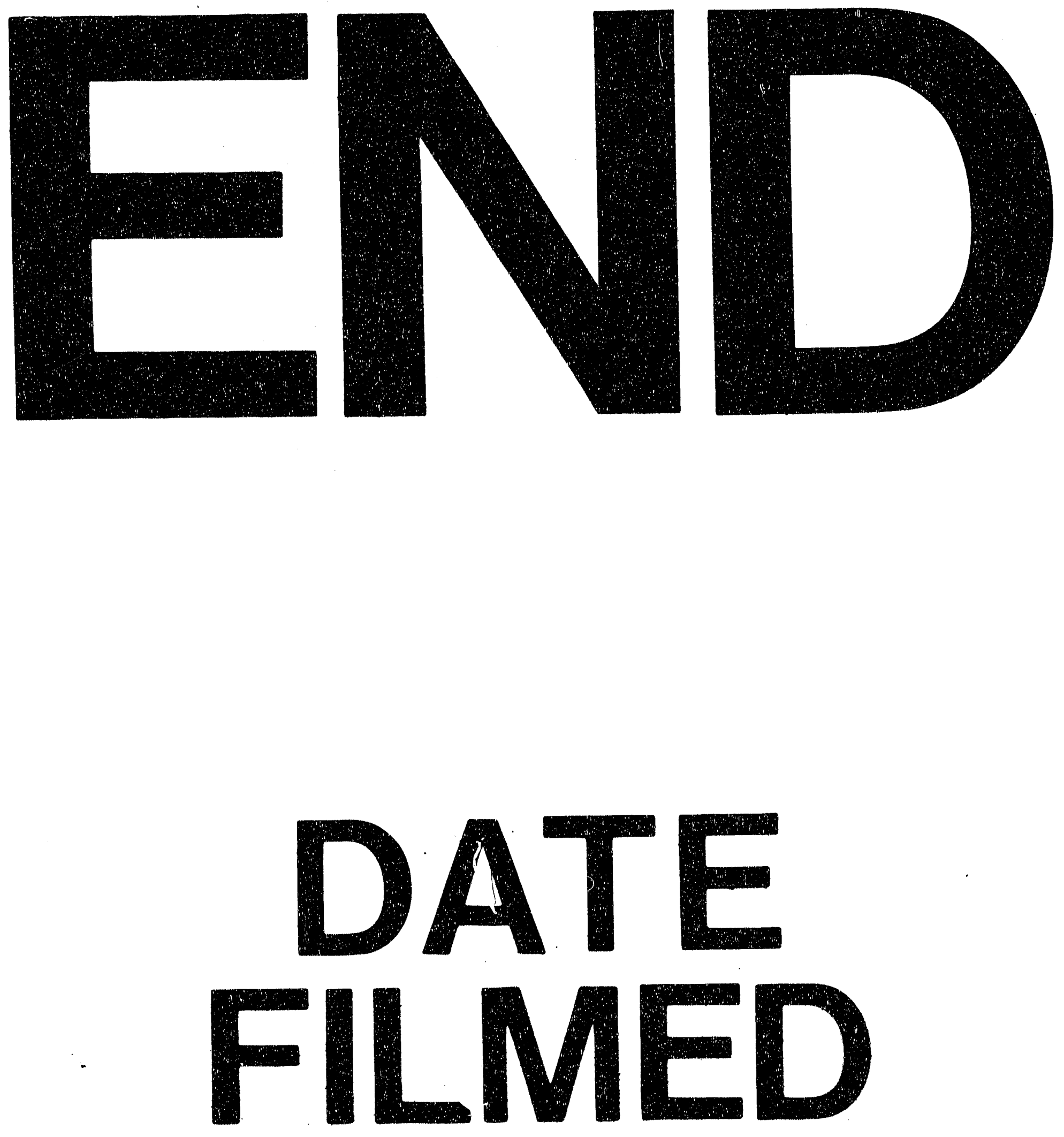

辛

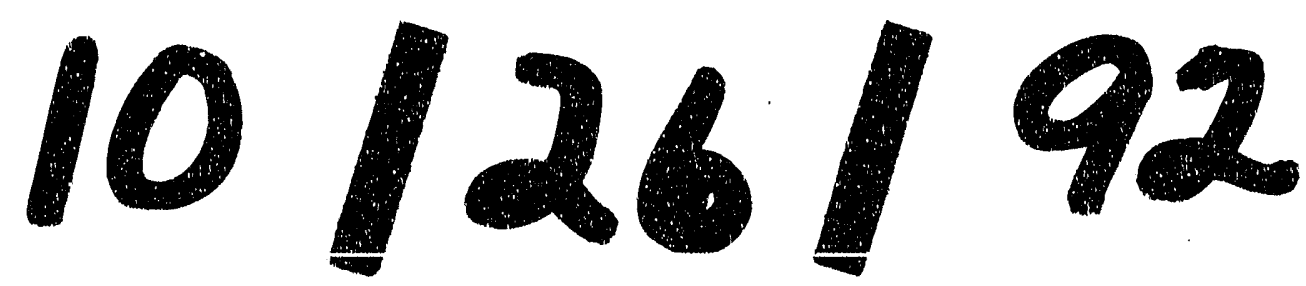


